A N N A L E S Annales de Bretagne et des Pays de l'Ouest

\title{
Les premiers temps chrétiens dans le territoire de la
} France actuelle

\section{Yves Henigfeld}

\section{(2) OpenEdition}

1 Journals

Édition électronique

URL : http://journals.openedition.org/abpo/2179

DOI : $10.4000 / a b p o .2179$

ISBN : 978-2-7535-1841-4

ISSN : 2108-6443

Éditeur

Presses universitaires de Rennes

Édition imprimée

Date de publication : 30 décembre 2011

Pagination : 127-131

ISBN : 978-2-7535-1839-1

ISSN : 0399-0826

\section{Référence électronique}

Yves Henigfeld, "Les premiers temps chrétiens dans le territoire de la France actuelle », Annales de Bretagne et des Pays de l'Ouest [En ligne], 118-4 | 2011, mis en ligne le 30 décembre 2013, consulté le 23 septembre 2020. URL : http://journals.openedition.org/abpo/2179 ; DOI : https://doi.org/10.4000/ abpo. 2179

Ce document a été généré automatiquement le 23 septembre 2020

(c) Presses universitaires de Rennes 


\title{
Les premiers temps chrétiens dans le territoire de la France actuelle
}

\author{
Yves Henigfeld
}

\section{RÉFÉRENCE}

PARIs-Poulain, D., ISTRIA, D., NARdi CombescuRe, S. (dir.), Les premiers temps chrétiens dans le territoire de la France actuelle. Hagiographie, épigraphie et archéologie : nouvelles approches et perspectives de recherches, Actes du colloque international d'Amiens, Université de Picardie Jules Vernes, Faculté des Arts, 18-20 janvier 2007, Rennes, PUR, coll.

« Archéologie \& Culture », 2009, 252 p., ill.

1 Cet ouvrage de 252 pages correspond à la publication des actes d'un colloque international intitulé Les premiers temps chrétiens dans le territoire de la France actuelle. Hagiographie, épigraphie et archéologie : nouvelles approches et perspectives de recherches, qui s'est tenu à l'Université de Picardie, en janvier 2007. Publié sous la direction de Dominique Paris Poulain, Daniel Istria et Sara Nardi Combescure, l'ouvrage regroupe près d'une vingtaine de contributions répartie dans trois sections thématiques.

2 La première partie, intitulée Les premiers temps chrétiens: l'apport des sources historiques, hagiographiques et épigraphiques (p.7-82), s'ouvre par une étude dans laquelle Brigitte Meijns et Charles Mériaux (p. 19-33) revisitent le cycle hagiographique de Rictiovar, afin d'apporter un éclairage sur la topographie chrétienne de la province de Reims. L'analyse est intentionnellement limitée à six basiliques martyriales rurales (Bazoches, Fismes, Sains, Saint-Just, Saint-Quentin et Seclain).

Dans une seconde contribution, Luce Pietri (p.35-40) s'interroge sur la place d'évangélisateur et le parcours singulier de l'ascète stylite Vulfilaicus, que Grégoire de Tours a rencontré en 585 à Eposium (Carignan) dans les Ardennes. Ce texte est suivi par une réflexion de Bruno Dumézil sur le rôle de Childebert $\mathrm{I}^{\mathrm{er}}$ dans la christianisation des Gaules (p. 41-49), puis par une étude de Sara Nardi Combescure (p.51-57) sur la 
signification religieuse et sociale des coupes en verre à décor chrétien du $\mathrm{IV}^{\mathrm{e}}$ siècle d'Homblières et de Vermand dans l'Aisne, représentant respectivement des épisodes de l'Ancien testament (Adam et Ève et l'arbre du Paradis, Daniel et les lions, Suzanne et les vieillards et Daniel et le Dragon de Bel) et la résurrection de Lazare. Dans la même perspective, Stéphanie Lambot propose un (ré)examen de vingt-trois objets «épigraphiques " provenant pour l'essentiel de Belgique, mais aussi du nord de la France, en s'interrogeant sur le degré de croyance de leurs possesseurs (p. 59-69). Il s'agit en l'occurrence de seize coupes en verres ornées d'un chrisme, de trois céramiques en pseudo-sigillée d'Argonne (Chenet 186) ornées à la molette, de trois bagues et d'une plaque-boucle de ceinture. Cette première partie s'achève par une relecture, par Françoise Prévot, de la place du célèbre évêque Didier de Cahors, dans la construction de la cité chrétienne de 630 à 655 (p. 71-82). L'auteur propose notamment d'attribuer la rédaction de la Vita Desiderii à une date immédiatement postérieure à la mort de Didier, l'objectif du biographe étant de valoriser l'œuvre de l'évêque constructeur, tant sur le plan matériel que spirituel.

4 La deuxième section, intitulée La topographie des cités chrétiennes et des agglomérations secondaires. Basiliques, baptistères et groupes épiscopaux : l'apport récent de l'archéologie, constitue l'essentiel de l'ouvrage (p. 83-202). Elle commence par une contribution de Marc Heijmans (p. 85-98), qui porte sur l'église paléochrétienne de l'enclos Saint-Césaire à Arles (Bouches-du-Rhône). L'auteur présente les résultats de la première année de fonctionnement d'un programme collectif de recherches et de plusieurs interventions archéologiques, menées entre 2003 et 2006. Ces dernières ont révélé la présence d'une abside de $20 \mathrm{~m}$ de diamètre à l'ouverture, incluant une seconde abside, interprétée comme les vestiges d'un synthronos ou banc presbytéral, mais qui pourrait peut-être aussi correspondre à une abside plus ancienne. Dans un addendum, l'auteur précise que des interventions complémentaires, réalisées en 2007 et 2008, ont permis de compléter le plan du sanctuaire, dont la construction est attribuée à la première moitié du vi ${ }^{e}$ siècle.

5 Dans la contribution suivante (p.99-118), Manuel Moliner dresse un tableau de la topographie religieuse de Marseille, en évoquant les principales découvertes archéologiques qui ont ponctué l'histoire de la recherche, depuis les premiers travaux d'envergure engagés au XIX ${ }^{\mathrm{e}}$ siècle, jusqu'à la découverte spectaculaire, en 2003-2004, de la basilique suburbaine et funéraire de la rue Malaval. L'abside semi-circulaire de ce sanctuaire abritait notamment un autel recouvrant un caisson de reliques et une tombe privilégiée, interprétée comme une Memoria, à l'origine d'un phénomène d'inhumations ad sanctos.

6 Un autre ensemble urbain, lui aussi fouillé en 2003-2004 à l'occasion d'une opération préventive, est présenté par Frank Gabayet (p. 119-135). Il s'agit de l'espace résidentiel du quartier épiscopal paléochrétien de Valence, édifié au ve siècle dans un secteur densément urbanisé depuis le $\mathrm{I}^{\text {er }}$ siècle de notre ère, au sud de l'actuelle cathédrale Saint-Apollinaire. Outre la présence de bâtiments ouverts sur des cours, la fouille a révélé un bel ensemble thermal et un édifice à abside, correspondant vraisemblablement à une chapelle. À partir $\mathrm{du} \mathrm{VI}^{\mathrm{e}}$-VII ${ }^{\mathrm{e}}$ siècle, la topographie du secteur se modifie sensiblement, avec la destruction des balnéaires et la construction de deux nouvelles églises, dont la plus récente est attribuée aux $\mathrm{x}^{\mathrm{e}}$-XII ${ }^{\mathrm{e}}$ siècles.

7 Ces trois présentations sont suivies par une synthèse de Marc Guyon (p. 137-145) sur la topographie des cités du Midi de la Gaule, envisagée à la lumière des résultats de la 
vaste enquête sur la Topographie chrétienne des cités de la Gaule, dont la publication arrive à son terme. L'auteur évoque en particulier les 74 villes épiscopales identifiées dans le Midi de la Gaule, pour lesquelles les données archéologiques restent inégales, voire inexistantes pour un quart d'entre elles. Il étend son propos aux agglomérations secondaires, puis aux sites ruraux, qui, probablement très tôt, avant le tournant $d u v^{e}$ siècle, se dotent d'édifices de cultes et parfois de baptistères, construits sur le modèle des ensembles urbains, et qui montrent que l'étude des premiers temps chrétiens ne peut être réduite aux seuls chefs-lieux de cité.

Cette présentation synthétique est suivie par cinq études de cas, respectivement consacrées au site de l'église majeure de Saint-Quentin (Aisne), au groupe épiscopal d'Ajaccio (Corse-du-Sud), au baptistère de Limoges (Haute-Vienne), à la basilique des Champs Saint-Martin de Rezé (Loire-Atlantique) et au baptistère de Roanne (Loire). Dans sa contribution intitulée "Autour de la tombe de Saint-Quentin, 1864-2004» (p. 147-160), Christian Sapin revient dans le détail sur les textes et sur les recherches entreprises sur le site de l'ancienne collégiale de Saint-Quentin depuis la seconde moitié du XIX ${ }^{\mathrm{e}}$ siècle, notamment marquées par les travaux de P. Bénard en 1864, puis d'E. Will en 1955. Une fouille programmée, qu'il a conduite de 2004 à 2007, lui offre l'occasion de renouveler la documentation et de réinterpréter les vestiges observés anciennement. Après une probable occupation antique et des aménagements en relation avec la tombe du saint datant de l'Antiquité tardive (état 2), un lieu de culte et des sépultures ad sanctos sont attestés au $\mathrm{VI}^{\mathrm{e}}$ siècle (état 3). À cet aménagement succède un nouvel édifice à chevet plat, construit au viI ${ }^{\mathrm{e}}$ siècle, au moment où l'on déplace la tombe du saint derrière l'autel (état 4). À l'époque carolingienne (état 5), on construit une abside et une crypte annulaire, dont le plan est modifié au xIII ${ }^{e}$ siècle.

Le groupe épiscopal d'Ajaccio a été découvert à l'occasion d'une fouille préventive conduite par Daniel Istria en 2005, à une dizaine de mètres au sud de la cathédrale médiévale, cette dernière ayant été détruite à l'époque moderne (p.161-173). Les vestiges mis au jour correspondent à un baptistère, dont la construction est attribuée au début $\mathrm{du} \mathrm{vI}^{\mathrm{e}}$ siècle. La partie dégagée correspond à une abside abritant une cuve baptismale de plan cruciforme allongée, dont le volume a été progressivement réduit dans le courant du haut Moyen Âge de façon à en faciliter l'accès, au point d'être finalement remplacé par une cuve cylindrique insérée dans une nouvelle église. Autour des années 600 , la construction de ce bâtiment entraîne des aménagements funéraires et coïncide avec le développement, à l'est de l'édifice, d'un espace artisanal et agricole.

Un autre baptistère a été mis en évidence lors d'un sondage effectué en 2004, devant le portail nord de la cathédrale de Limoges. Cette opération a donné lieu, en 2005 et 2006, à une fouille programmée, dont les résultats sont présentés par Julien Denis (p. 175-180). Établi dans un secteur déjà urbanisé au IV siècle, ce vaste édifice $\left(290 \mathrm{~m}^{2}\right)$ de plan rayonnant est constitué d'une pièce hexagonale abritant une piscine circulaire, se prolongeant par six pièces rectangulaires. Construit dans la première moitié du $v^{e}$ siècle, le bâtiment a fait l'objet de remaniements au $\mathrm{VI}^{\mathrm{e}}$ siècle, avec l'ajout d'une abside et d'un autel. L'ensemble est détruit pour laisser place, au début du XIII ${ }^{e}$ siècle, à une petite église paroissiale dédiée à Saint-Jean-Baptiste. Une partie de la discussion porte sur la chronologie de l'édifice qui repose, en grande partie, sur des datations $14^{\mathrm{C}}$.

11 La basilique des Champs Saint-Martin a, quant à elle, été découverte en 2001, dans l'agglomération portuaire et antique de Rezé (Ratiatum), établie, en fond d'estuaire sur la rive gauche de la Loire, légèrement à l'ouest de Nantes. Fouillé et présenté par Lionel 
Pirault (p.181-194), ce vaste bâtiment $\left(750 \mathrm{~m}^{2}\right.$ de surface restituée) correspond à un édifice à chevet plat comprenant une nef de 19,60 x $14 \mathrm{~m}$, ayant fait l'objet d'au moins deux remaniements. La nef, bordée par deux galeries externes de $4 \mathrm{~m}$ de large, se prolonge à l'est, par un chœur liturgique doté d'une base d'autel et pourvu de deux annexes latérales. Cet ensemble est attribué, sur la base d'indices céramologiques, au début $\mathrm{du} \mathrm{VI}^{\mathrm{e}}$ siècle. Outre son intérêt pour l'histoire de l'agglomération à l'époque tardo-antique, le site présente la particularité d'avoir livré un bel ensemble de fragments de verre à vitre.

Tout aussi inattendue fut la découverte, en 2005, d'un baptistère au centre de la vieille ville de Roanne, principalement connue pour son passé d'agglomération antique et de bourg castral. Présenté par Monique Le Nézet-Célestin (p.195-202), le baptistère est implanté dans le quartier sud de l'agglomération gallo-romaine. Il est plus précisément édifié à l'emplacement d'un bâtiment construit au $\mathrm{III}^{\mathrm{e}}$ siècle. L'installation primitive se présente sous la forme d'une cuve baptismale octogonale, établie dans une pièce excavée dallée de tegulae. Devenu trop exigüe, le baptistère est agrandi et pourvu à l'est d'une abside semi-circulaire, le sol est rehaussé et une nouvelle cuve baptismale, de plan circulaire, est aménagée. Cet ensemble est attribué, par comparaison et en l'absence d'autres indices de datation, à la fin du $v^{e}$ siècle ou au vi ${ }^{e}$ siècle. Vers le $x^{e}$ ou le début $\mathrm{du} \mathrm{XI}^{\mathrm{e}}$ siècle, le secteur change de fonction, comme l'atteste la découverte d'une aire d'ensilage.

La troisième section intitulée Christianisme en milieu rural (p. 203-247) s'ouvre par une contribution collective que l'on doit à Isabelle Catteddu, Florence Carré, François Gentili, François Delahaye, Jean-Yves Langlois et Pascal Couanon (p. 205-228), dans laquelle les auteurs présentent une série de fouilles d'églises rurales du haut Moyen Âge récemment découvertes dans le nord de la France. C'est l'occasion pour les auteurs de dresser un premier état de la question et d'amorcer une réflexion sur la précocité de la relation entre l'église et le cimetière en milieu rural. Certains de ces sites, fouillés de façon extensive et exhaustive sur de vastes superficies, sont associés à un habitat. C'est le cas notamment de Saleux (Picardie) de Mondeville (Calvados) de Tournedos-surSeine (Seine-Maritime) ou de Serris (Seine-et-Marne). D'autres sites correspondent à des découvertes plus ponctuelles, localisées en Normandie, comme les églises de NotreDame-de-Bondeville (Seine-Maritime) ou de Thaon (Calvados). Souvent attestées dès le $\mathrm{VII}^{\mathrm{e}}$ siècle, ces églises rurales jouent un rôle déterminant dans l'organisation de l'espace et même si l'échantillonnage reste encore réduit - pas plus d'une dizaine de sites pour de nord de la France - ces résultats montrent la diversité de ces édifices ruraux, qui posent des problèmes d'interprétation, tant sur le plan chronologique que fonctionnel.

Ce texte est suivi par deux contributions de synthèse sur les premiers temps chrétiens dans le sud-ouest de la France, respectivement proposées par Christine Delaplace (p. 229-233) et Marie-Geneviève Colin (p. 235-247). Dans la première, l'auteur revient sur les enseignements d'un colloque publié en 2005, organisé à Toulouse en 2003, qui portait sur les origines de la paroisse en Gaule méridionale $d u v^{e}$ au $I^{e}$ siècle. La seconde a pour thème les campagnes de Novempopulanie du $I v^{e}$ au $x^{e}$ siècle. Le tout s'achève par un chapitre conclusif (p. 249-252), dans lequel Brigitte Beaujard esquisse un bilan de ces trois journées d'étude.

Si la question des premiers temps chrétiens n'est pas en soit une thématique véritablement nouvelle, la nature et la qualité de la plupart des contributions présentée dans cet ouvrage collectif montre que la recherche est plus que jamais active. La 
documentation ancienne est sans cesse réinterrogée et la documentation récente, principalement archéologique, ouvre des perspectives stimulantes sur la place des édifices religieux dans des agglomérations secondaires et sur l'apparition précoce des églises sur des sites d'habitat ruraux du haut Moyen Âge.

Il est toutefois dommage que les textes proposés soient parfois desservis par des illustrations de qualité inégale. Certaines photos ou certains plans sont illisibles ou présentés dans des formats inadaptés, et auraient sans doute gagné en lisibilité s'ils avaient été retravaillés ou présentés en couleur. Ceci étant dit, et pour expliquer en partie ces quelques imperfections de forme, il convient de souligner le peu de temps écoulé entre le colloque et la parution de l'ouvrage (2 ans).

Parmi les éléments saillants de ce colloque, on insistera surtout sur la place importante accordée à l'archéologie préventive, qui, à côté des fouilles programmées, a sensiblement contribué à renouveler, depuis une vingtaine d'années, la perception de la christianisation de la Gaule, que ce soit en milieu rural ou urbain. La publication de ces actes offre l'occasion de faire connaître des sites parfois inédits ou méconnus, d'amorcer des synthèses originales et d'ouvrir de nouvelles pistes de recherche. En ce sens, le parti pris des organisateurs de réserver une place de choix aux apports récents de l'archéologie mérite d'être salué.

Yves Henigfeld 